\author{
Safety Commission
}

\title{
Monte Carlo simulation of muon-induced background of an anti-Compton gamma-ray spectrometer placed in a surface and underground laboratory
}

\author{
P. Vojtyla ${ }^{\mathrm{a}}$ and P. Povinec ${ }^{\mathrm{b}}$ \\ a) CERN, Safety Commission, CH-1211 Geneva 23, Switzerland \\ b) IAEA, Marine Environmental Laboratory, Monte Carlo, MC-98012, Monaco
}

\begin{abstract}
Simulations of cosmic ray muon induced background of an HPGe detector placed inside an anti-Compton shield on the surface and in shallow underground is described. Investigation of several model set-ups revealed some trends useful for design of low-level gamma-ray spectrometers. It has been found that background spectrum of an HPGe detector can be scaled down with the shielding depth. No important difference is observed when the same set-up of the anti-Compton spectrometer is positioned horizontally or vertically. A cosmic-muon rejection factor of at least 40 (at around $1 \mathrm{MeV}$ ) can be reached when the anti-Compton suppression is operational. The cosmicmuon background can be reduced to such a level that other background components prevail, like those from the residual contamination of the detector and shield materials and/or from radon, especially for the underground facilities.
\end{abstract}

Presented at the International Conference on Isotopes in Environmental Studies,

Aquatic Forum 2004, 25-29 October 2004, Monaco

Submitted for publication in a special issue of Elsevier's Environmental

Radioactivity Book Series. 


\section{Introduction}

The background of low-level HPGe spectrometers placed on the surface or in shallow shielding depths is dominated by the cosmic-muon induced component (Heusser, 1995). Present Monte Carlo detector simulation tools, such as GEANT developed at CERN (1990), are capable to deal with the simulation of particle passage and detectors in complex systems, and the necessary computing power is available. Hence, there has been an opportunity to examine the influence of various parameters on the detector background, to investigate the trends in model systems or even to compute the cosmic-muon induced background before a lowlevel system was constructed.

Vojtyla et al. (1994, 1995) developed and validated Monte Carlo codes based on GEANT that were suitable for simulating the cosmic-muon background component in typical HPGe gamma-ray spectrometers placed on the surface. The core of the code was a standard cosmic muon particle generator simulating the actual momentum and direction distributions of cosmic-muons at sea level. With this tool, the physics governing the background induction process could be understood quantitatively and the influence of various parameters on the background, mainly the effects of the shield material and the shield dimensions, could be investigated easily.

Lower cosmic-muon background is obtained in tighter shields made of lead lined from the inner side with as thin layers of low- $Z$ materials as possible (Vojtyla, 1996). It was a logical step to extend the models to shallow shielding depths, not deeper than about 50 metres water equivalent (m w.e.) that was done by Vojtyla and Povinec (2000).

This work focuses on even more complex systems - anti-Compton spectrometers - and describes investigations carried out for the underground facility of the IAEA's Marine Environment Laboratory (IAEA-MEL) in Monaco. A model detector was considered and the work aimed at revealing important trends in the background characteristics.

\section{Simulation codes}

\subsection{Surface laboratories}

The details of the simulation Monte Carlo code for surface laboratories have already been described by Vojtyla (1995), here we recall just some essential points. HPGe detectors are described in GEANT (CERN, 1990) complemented with a standard sea-level cosmic-muon random initial-particle generator. The set-ups are placed entirely in fictive volumes of cylindrical or rectangular shapes, which fit them as closely as possible. Surface areas visible from above are bombarded with cosmic-muons that have suitable momentum, charge and angular distributions. It is assumed that the differential muon flux $j(\vartheta, p)$, where $\vartheta$ is the zenith angle and $p$ is the muon momentum, can be expressed as $j(\vartheta, p)=j(0, p) \cos ^{n(p)} \vartheta$.

The data for constructing the functions $j(0, p)$ and $n(p)$ can be found in the literature (Allkofer et al., 1967, 1971, 1975, 1984; Moroney and Parry, 1954). It was shown that finer dependencies such as the EastWest muon flux asymmetry, the dependence of the muon charge ratio on the muon momentum, latitudinal and seasonal variations can be neglected (Vojtyla, 1995).

The simulation processes for the calculation of the HPGe detector background have included (in the order of importance for the background induction): (i) production of $\delta$-electrons by muons, and their bremsstrahlung; (ii) direct electron-positron pair production by muons; (iii) muon decay; (iv) muon bremsstrahlung; (v) direct ionization by muons in the sensitive volume of the HPGe detector; (vi) others.

\subsection{Underground laboratories}

The energy losses of muons below about $500 \mathrm{GeV} / \mathrm{c}$ are mostly due to quasi-continuous ionisation. For muons of higher energies, discrete processes dominate (bremsstrahlung, fast muon nuclear interactions). The transport of muons in the lower energy range is simpler, as the muons lose their energy almost continuously and they do not change their directions significantly. In the higher energy range, significant fluctuations of both energy and direction can occur. If the prevailing part of the muon spectrum observed underground stems mostly from muons with original energies below a few hundreds of $\mathrm{GeV}$, the attenuation of the muon flux can be taken into account simply by accounting for mean ranges and integrated energy losses of muons. Deep underground, where the muon spectrum originates from sea-level muons of $1 \mathrm{TeV} / \mathrm{c}$ or more, this approach is not possible. The case of shallow shielding depths can be treated therefore in a simpler way.

For generic set-ups when the accurate topography and the material definition of the overburden rock are not specified, we have to limit ourselves only to coarse estimates of the background. This allows us to accept two approximations:

(i) there are no fluctuations in muon ranges and energy losses in the rock;

(ii) there is no deviation from the original muon direction due to multiple scattering to small angles.

Although the effects (i) and (ii) take place in reality, they can be ignored in calculations of the muon transport underground, as they are partly smoothed-out due to continuous energy and angle spectra of cosmic muons.

The muon generator in the codes for the simulating muon-induced background under an overburden is similar to that at the sea level, but the muon rejection and momentum reduction algorithms are applied. After the generation of the initial kinematics of the muon has 
been completed, the range of a muon in rock is calculated and compared with the slanted depth of the counting system $X=D / \cos \vartheta$, where $D$ is the vertical depth and $\vartheta$ is the muon zenith angle. If the range is shorter than the slanted depth, the event is rejected. Otherwise the original muon momentum $p_{0}$ is reduced according to the formula

$p_{\text {red }}=p_{0}-R^{-1}(X)$,

where $R^{-1}(X)$ is the inverse function of a function describing range of muons in dependence on the muon momentum $R(p)$. It is assumed that the rock has the "standard rock" composition with the density of $2.65 \mathrm{~g} \cdot \mathrm{cm}^{-3}$, the average mass number $A=22$ and the average atomic number $Z=11$ (Heusser, 1995). The transport of muons through the standard rock was simulated using GEANT and data on mean ranges for various muon momenta have been in agreement with observations (Vojtyla and Povinec, 2000).

The rejection method is not time consuming, as most of the total computing time is spent for particle tracking. It wastes only random number generator. For example, for an overburden of $30 \mathrm{~m}$ w.e., about $80 \%$ of muons are rejected. The method is in principle applicable to any complex shapes above the underground laboratory, such as hilly terrain or buildings; however, the rejection routine becomes more complicated. The assumed muon momentum range of $0.2-100 \mathrm{GeV} / \mathrm{c}$ is sufficient for shallow shielding depths.

\section{Model detector system}

An n-type coaxial $100 \%$ relative efficiency (in comparison with $7.5 \mathrm{~cm} \times 7.5 \mathrm{~cm} \mathrm{NaI}(\mathrm{Tl})$ detector) HPGe detector was assumed to be surrounded by a model $\mathrm{NaI}(\mathrm{Tl})$ anti-Compton shield and the whole assembly was placed inside a passive shield on the surface or 30 m w.e. underground. The HPGe detector corresponded to a real design. The anti-Compton shield consisted of an annular $\mathrm{NaI}(\mathrm{Tl})$ crystal with the inner diameter of $11 \mathrm{~cm}$, the outer diameter of $30 \mathrm{~cm}$ and the height of $40 \mathrm{~cm}$, closed from one side by another cylindrical $\mathrm{NaI}(\mathrm{Tl})$ crystal $7.5 \mathrm{~cm}$ diameter $\times 7.5 \mathrm{~cm}$ height.

The whole detector set-up was placed inside a cylindrical shield made of $15 \mathrm{~cm}$ thick lead with the diameter of $70 \mathrm{~cm}$ and length of $90 \mathrm{~cm}$. The HPGe detector head was so deep in the anti-Compton shield that only free space of $11 \mathrm{~cm}$ in diameter and $5 \mathrm{~cm}$ in height was allowed for samples. Obviously, the antiCompton shield reduces not only the Compton continuum but also the cosmic-muon induced background. The reduction is very efficient due to the high detection efficiency of $\mathrm{NaI}(\mathrm{Tl})$ crystals for various radiation types.

Practically there are two possibilities to position the whole anti-Compton system: either vertically (the $\mathrm{NaI}(\mathrm{Tl})$ stopcock is on the top), or horizontally. A drawing of the set-up positioned horizontally is shown in Fig. 1. It cannot be assessed simply what configu- ration would be more efficient in respect to the background reduction by the anti-Compton shield. Therefore simulations of the vertical and horizontal set-ups using the developed code were carried out.

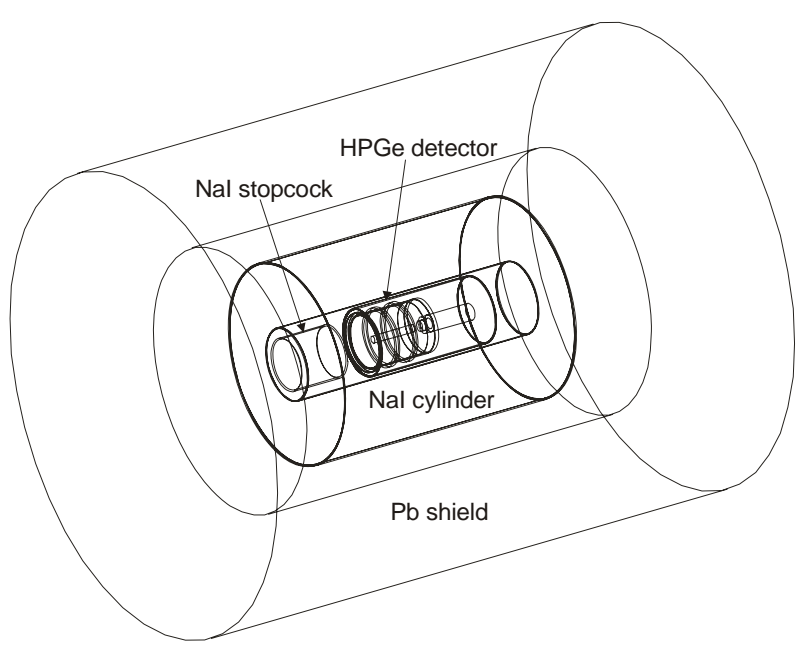

Figure 1: Drawing of the anti-Compton set-up positioned horizontally.

The code for the horizontal set-up has a more complicated muon generator than the standard codes described earlier. A box containing the passive shield is bombarded by cosmic-muons instead of horizontally positioned cylinder, allowing using the already written and debugged functions for kinematics distributions. Because the basis of the box is not a square, bombardment of sides of different areas has to be correctly sampled so that the impact rates are proportional to the respective areas.

\section{Results and discussion}

The results for the vertical and horizontal anti-Compton shield arrangements on sea level and underground are summarized in Tables 1 and 2, respectively. The corresponding lower energy spectra are shown in Figs. 2 and 3. The simulated background spectra $30 \mathrm{~m}$ w.e. underground show the same features as those at sea level, but all absolute background characteristics are reduced by a general factor of about 3 that was observed also in passive set-ups in the previous work of Vojtyla and Povinec (2000). The background spectra in the energy region up to $100 \mathrm{MeV}$ are shown in Fig. 4.

A striking feature of the background spectra is that the difference between the horizontal and vertical arrangements is negligible in the lower energy region. This can be caused by equilibration of the lowerenergy photon flux inside the shield cavity and antiCompton $\mathrm{NaI}(\mathrm{Tl})$ crystals. The vertical arrangement seems to be slightly better than the horizontal one below about $1000 \mathrm{keV}$. A mistake during writing or running the codes is excluded because a comparison of the spectra in the higher energy region in Fig. 4 shows that the spectra differ significantly in the region above about $10 \mathrm{MeV}$ where the contribution of the secondary processes in the shields is negligible, and the direct ionisation by muons prevails. 
Table 1: Background characteristics of HPGe detectors with anti-Compton shields in horizontal and vertical positions simulated at sea level.

\begin{tabular}{|c|c|c|c|c|c|c|}
\hline Anti-Compton off & \multicolumn{3}{|c|}{ Horizontal } & \multicolumn{3}{|c|}{ Vertical } \\
\hline $0-3000 \mathrm{keV}\left[\mathrm{min}^{-1}\right]$ & 89.5 & \pm & 0.1 & 90.1 & \pm & 0.1 \\
\hline $511 \mathrm{keV}\left[\mathrm{d}^{-1}\right]$ & 3090 & \pm & 30 & 3010 & \pm & 30 \\
\hline \multicolumn{7}{|l|}{ Anti-Compton on } \\
\hline $0-3000 \mathrm{keV}\left[\mathrm{min}^{-1}\right]$ & 1.04 & \pm & 0.01 & 0.914 & \pm & 0.012 \\
\hline $511 \mathrm{keV}\left[\mathrm{d}^{-1}\right]$ & 31 & \pm & 3 & 25 & \pm & 2 \\
\hline \multicolumn{7}{|l|}{ Reduction factor } \\
\hline $0-3000 \mathrm{keV}$ & 86 & \pm & 1 & 99 & \pm & 1 \\
\hline $511 \mathrm{keV}$ & 99 & \pm & 10 & 119 & \pm & 12 \\
\hline
\end{tabular}

Table 2: Background characteristics of HPGe systems with anti-Compton shields in the horizontal and vertical positions simulated $30 \mathrm{~m}$ w.e. underground.

\begin{tabular}{lccccccc}
\hline & \multicolumn{3}{c}{ Horizontal } & \multicolumn{5}{c}{ Vertical } \\
\hline Anti-Compton off & & & & & & & \\
$0-3000 \mathrm{keV}\left[\mathrm{min}^{-1}\right]$ & 29.7 & \pm & 0.1 & 30.1 & \pm & 0.1 \\
$511 \mathrm{keV}\left[\mathrm{d}^{-1}\right]$ & 1050 & \pm & 30 & 1030 & \pm & 30 \\
\hline Anti-Compton on & & & & & & \\
$0-3000 \mathrm{keV}\left[\mathrm{min}^{-1}\right]$ & 0.33 & \pm & 0.01 & 0.29 & \pm & 0.01 \\
$511 \mathrm{keV}\left[\mathrm{d}^{-1}\right]$ & 8.7 & \pm & 2.5 & 5.1 & \pm & 1.9 \\
\hline Reduction factor & & & & & & \\
$0-3000 \mathrm{keV}$ & 90 & \pm & 3 & 102 & \pm & 4 \\
$511 \mathrm{keV}$ & 120 & \pm & 35 & 200 & \pm & 70 \\
\hline
\end{tabular}

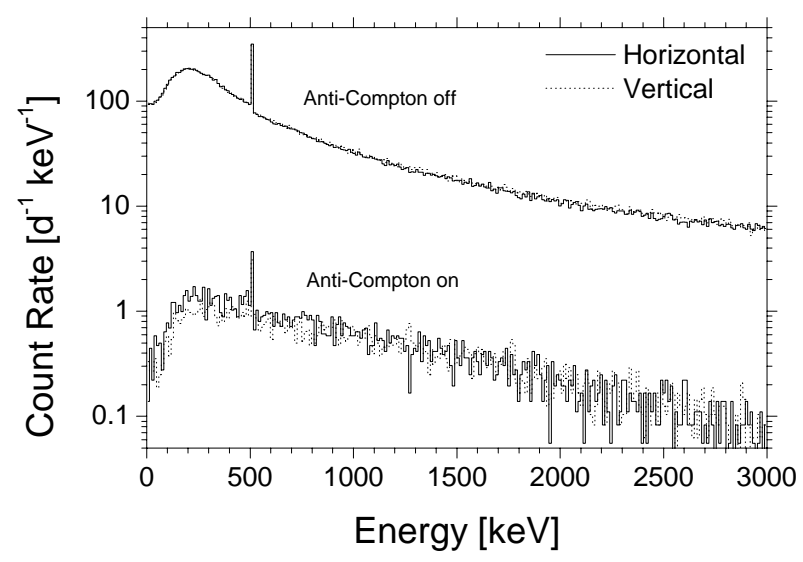

Figure 2: Lower-energy background spectra of the antiCompton gamma-ray spectrometer in the horizontal and vertical positions at sea level.

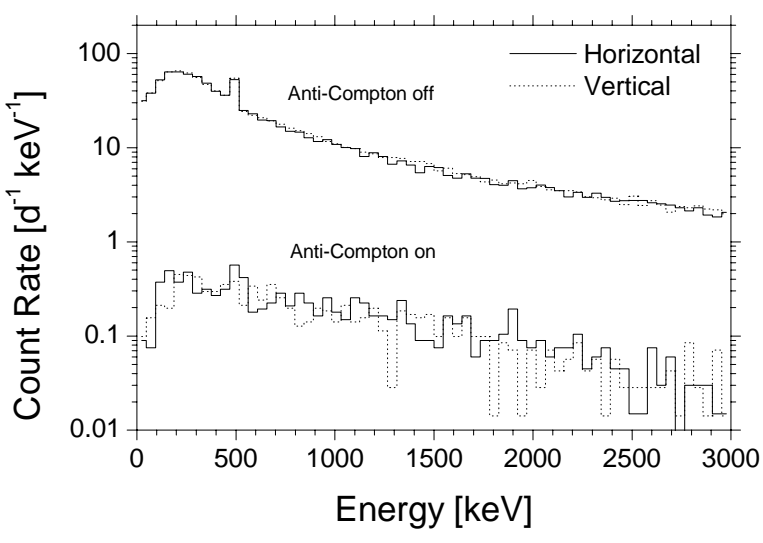

Figure 3: Lower-energy background spectra of the antiCompton spectrometer in the horizontal and vertical positions $30 \mathrm{~m}$.w.e. underground. 


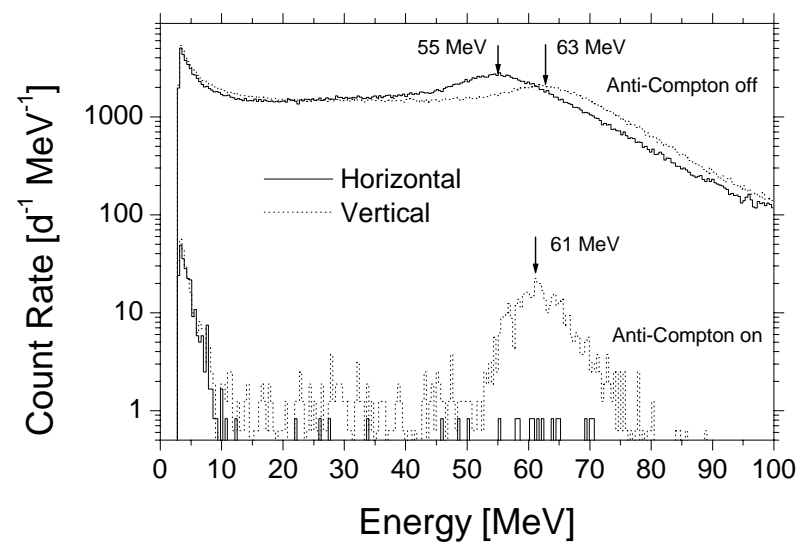

Figure 4: Higher-energy background spectra of the anti-Compton spectrometer in the horizontal and vertical positions at the sea level.

The wide peaks have maxima at different energies according to the track distributions inside the sensitive volume of the HPGe diode. With the anti-Compton rejection off, the maximum is reached at $55 \mathrm{MeV}$ for the horizontal position, corresponding to the most probable track length of $7.5 \mathrm{~cm}(7.3 \mathrm{MeV} / \mathrm{cm}$ ionisation losses of minimum ionising particles in Ge (Rev. Particle Properties, 1994)), which is close to the crystal diameter of $7.6 \mathrm{~cm}$. The muons moving in directions close to the vertical are likely to move along the HPGe crystal diameter. On the other hand, the maximum for the vertical position is reached at about $63 \mathrm{MeV}$ corresponding to the track length of $8.6 \mathrm{~cm}$, which is close to the height of the HPGe crystal of $8.2 \mathrm{~cm}$. The muons move more likely through the crystal height in this case. The apparent peak at $61 \mathrm{MeV}(8.4 \mathrm{~cm})$ observed for the vertical position with the anti-Compton rejection switched on stems from the "leakage" of muons through the space between the annular $\mathrm{NaI}(\mathrm{Tl})$ crystal and the stopcock $\mathrm{NaI}(\mathrm{Tl})$ crystal. A large number of muons must move in the direction parallel to the axis of the set-up to induce such a peak. This condition is well fulfilled in the vertical arrangement of the set-up when many low-energy muons move along the vertical direction.

Note that the presence of thick layers of $\mathrm{NaI}$ close to the detector crystal has a detrimental effect on the background continuum if the anti-Compton rejection is switched off. Although the arrangement could be considered as a small shield, the maximum in the continuum reaches about $200 \mathrm{~d}^{-1} \cdot \mathrm{keV}^{-1}$ on the surface to be compared, for example, with about $100 \mathrm{~d}^{-1} \cdot \mathrm{keV}^{-1}$ (at around $200 \mathrm{keV}$ ) for a small cylindrical descending- $Z$ lining shield (Vojtyla and Povinec, 2000), despite of the relatively high effective $Z$ and $A$ of $\mathrm{NaI}$ (46.6 and 111 , respectively). But this also implies that a thin housing of the NaI crystals, which was not assumed in the model set-up, would not have a considerable effect on the results.

The background reduction factor by the antiCompton rejection depends on the energy in the spectrum. Fig. 5 shows the background reduction factors calculated for various energies up to $3000 \mathrm{keV}$. It reaches values of about 200 for the lowest energies
(50-100 keV) and decreases down to about 40 at $1500 \mathrm{keV}$. Then it slowly increases up to about 100 at $3000 \mathrm{keV}$.

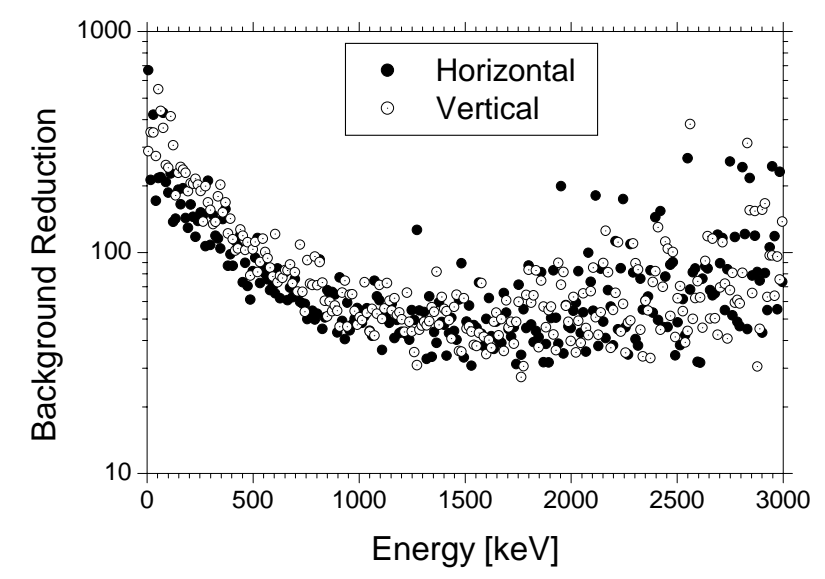

Figure 5: Dependence of the background induction factors of the anti-Compton suppression on the energy for the horizontal and vertical positions at sea level.

\section{Conclusions}

Monte Carlo simulations of cosmic-muon induced background in HPGe counting systems using strong background reduction techniques (underground, antiCompton shield) are feasible at present. Simulations of a model HPGe detector placed inside an anti-Compton shield on the surface and in shallow underground revealed some trends useful in design of such systems. First the scaling practice was confirmed. Given the detector set-up, its background spectra induced by cosmic-muons can be scaled down by a factor corresponding to the shielding depth. No important difference was observed when the same set-up was positioned horizontally or vertically.

An $\mathrm{NaI}(\mathrm{Tl})$ anti-Compton gamma-ray spectrometer inside a passive shield has a detrimental effect on the cosmic-muon induced background if the anti-Compton rejection is switched off because of a thick layer of a relatively low- $Z$ material, compared with standard shielding materials like lead.

On the other hand, a cosmic-muon rejection factor of at least 40 (at around $1.5 \mathrm{MeV}$ ) can be reached when the anti-Compton rejection is operational. In such situation, the cosmic-muon background is reduced to such a level that other background components prevail, like those from the residual contamination of the detector and anti-Compton shield materials, or from radon, especially for the underground facilities.

However, such high background reduction factors can be reached only if construction materials with negligible radionuclide contamination have been used for the construction of the HPGe detector, its cryostat, the $\mathrm{NaI}(\mathrm{Tl})$ detectors and the passive shielding and if radon is efficiently removed from the laboratory. 


\section{Acknowledgement}

IAEA-MEL operates under a bilateral agreement between the IAEA and the Government of the Principality of Monaco.

\section{References}

Allkofer, O.C. (1967). Die Höhen - und Zenitwinkelabhängigkeit der Energiespektren von Ultrastrahlungsteilchen. Fortschritte der Physik 15, 113-196.

Allkofer, O.C., Karstensen, K., Dau, W.D. (1971). The absolute cosmic ray muon spectrum at sea level. Phys. Lett. B36, 425-427.

Allkofer, O.C. (1975). Introduction to Cosmic Radiation. Thiemig, München.

Allkofer, O.C., Grieder, P.K.F. (1984). Cosmic Rays on Earth, Physics Data Nr. 25-1.

CERN (1990). GEANT Detector Description and Simulation Tool, CERN Program Library Office, CERN, Geneva, Switzerland.

Heusser, G. (1995). Low-radioactivity background techniques. Ann. Rev. Nucl. Part. Sci. 45, 543-590.

Moroney, I.R., Parry, I.K. (1954). Momentum distribution and charge ratio of $\mu$-mesons at zenith angles in the east-west plane. Austral. J. Phys. 7, 423-428.

Review of Particle Properties. (1994). Phys. Rev. D50, 1173.

Vojtyla, P., Beer, J., Št'avina, P. (1994). Experimental and simulated cosmic muon induced background of a Ge spectrometer equipped with a top side anticoincidence proportional chamber. Nucl. Instr. Meth. B86, 380-386.

Vojtyla, P. (1995). A computer simulation of the cosmic-muon background induction in a $\mathrm{Ge} \gamma$ spectrometer using GEANT. Nucl. Instr. Meth. B100, 87-97.

Vojtyla, P. (1996). Influence of shield parameters on cosmic-muon induced background of Ge $\gamma$-spectrometers. Nucl. Instr. Meth. B111, 163-170.

Vojtyla, P., Povinec, P.P. (2000). A Monte Carlo simulation of background characteristics of lowlevel HPGe detectors. Appl. Rad. Isotopes 53, 185-190. 